Erratum

\title{
Erratum to "The Effect of Acupuncture on the Motor Function and White Matter Microstructure in Ischemic Stroke Patients"
}

\author{
Yongxin Li, ${ }^{1}$ Ya Wang, ${ }^{1}$ Heye Zhang, ${ }^{2}$ Ping Wu, ${ }^{3}$ and Wenhua Huang ${ }^{1}$ \\ ${ }^{1}$ Institute of Clinical Anatomy, School of Basic Medical Sciences, Southern Medical University, Guangzhou 510515, China \\ ${ }^{2}$ Shenzhen Institutes of Advanced Technology, Chinese Academy of Sciences, Shenzhen 510855, China \\ ${ }^{3}$ The 3rd Teaching Hospital, Chengdu University of Traditional Chinese Medicine, Chengdu, Sichuan 610075, China \\ Correspondence should be addressed to Ping Wu; 19852021@cdutcm.edu.cn and Wenhua Huang; huangwenhua2009@139.com \\ Received 18 January 2016; Accepted 16 March 2016
}

Copyright (C) 2016 Yongxin Li et al. This is an open access article distributed under the Creative Commons Attribution License, which permits unrestricted use, distribution, and reproduction in any medium, provided the original work is properly cited.

In the article titled "The Effect of Acupuncture on the Motor Function and White Matter Microstructure in Ischemic Stroke Patients" [1], Ping Wu and Wenhua Huang contributed equally as corresponding authors.

\section{References}

[1] Y. Li, Y. Wang, H. Zhang, P. Wu, and W. Huang, "The effect of acupuncture on the motor function and white matter microstructure in ischemic stroke patients," Evidence-Based Complementary and Alternative Medicine, vol. 2015, Article ID 164792, 10 pages, 2015. 


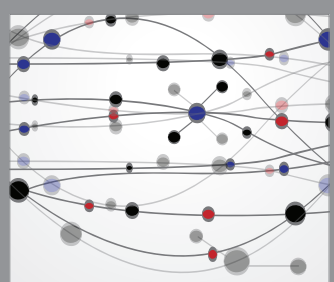

The Scientific World Journal
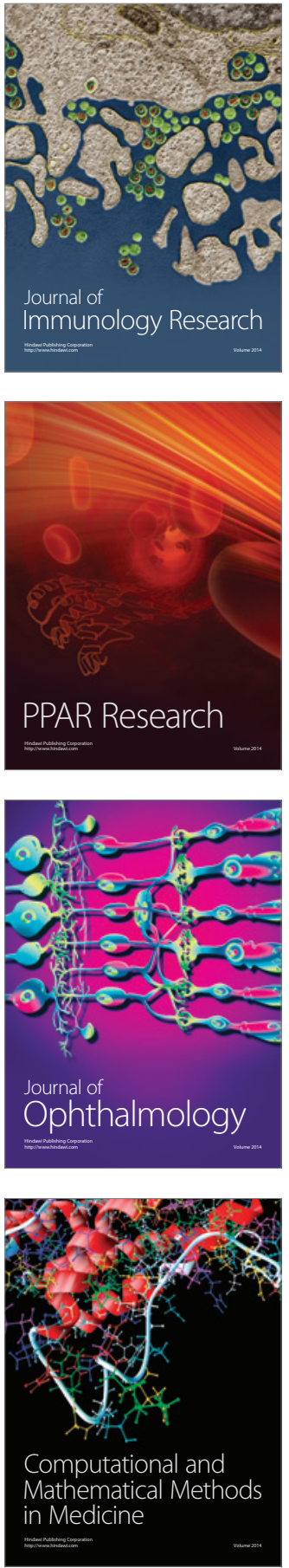

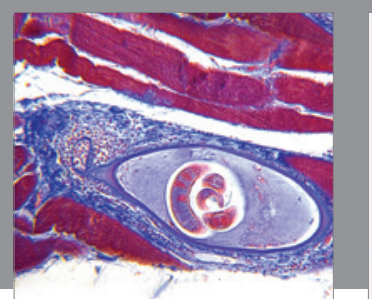

Gastroenterology Research and Practice

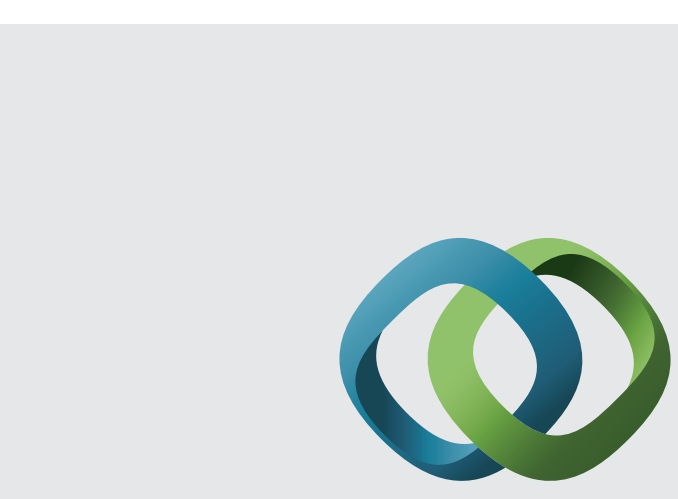

\section{Hindawi}

Submit your manuscripts at

http://www.hindawi.com
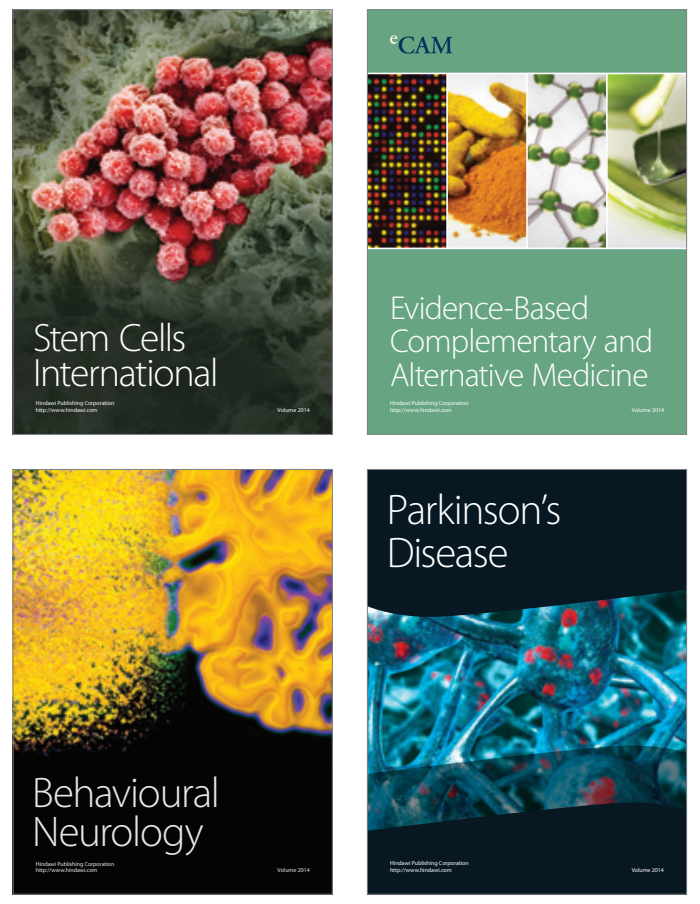
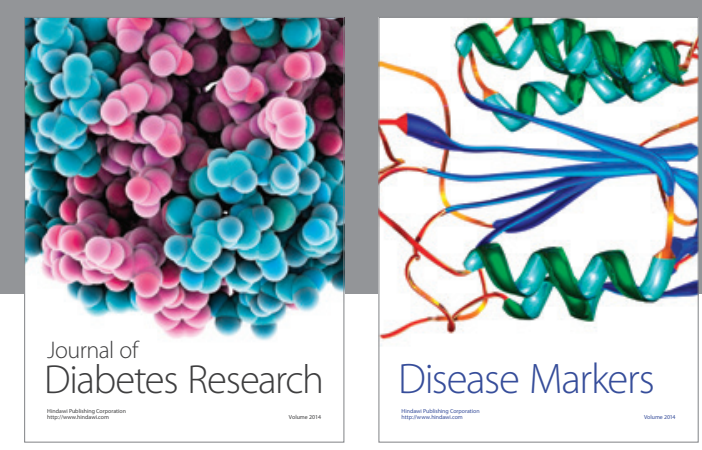

Disease Markers
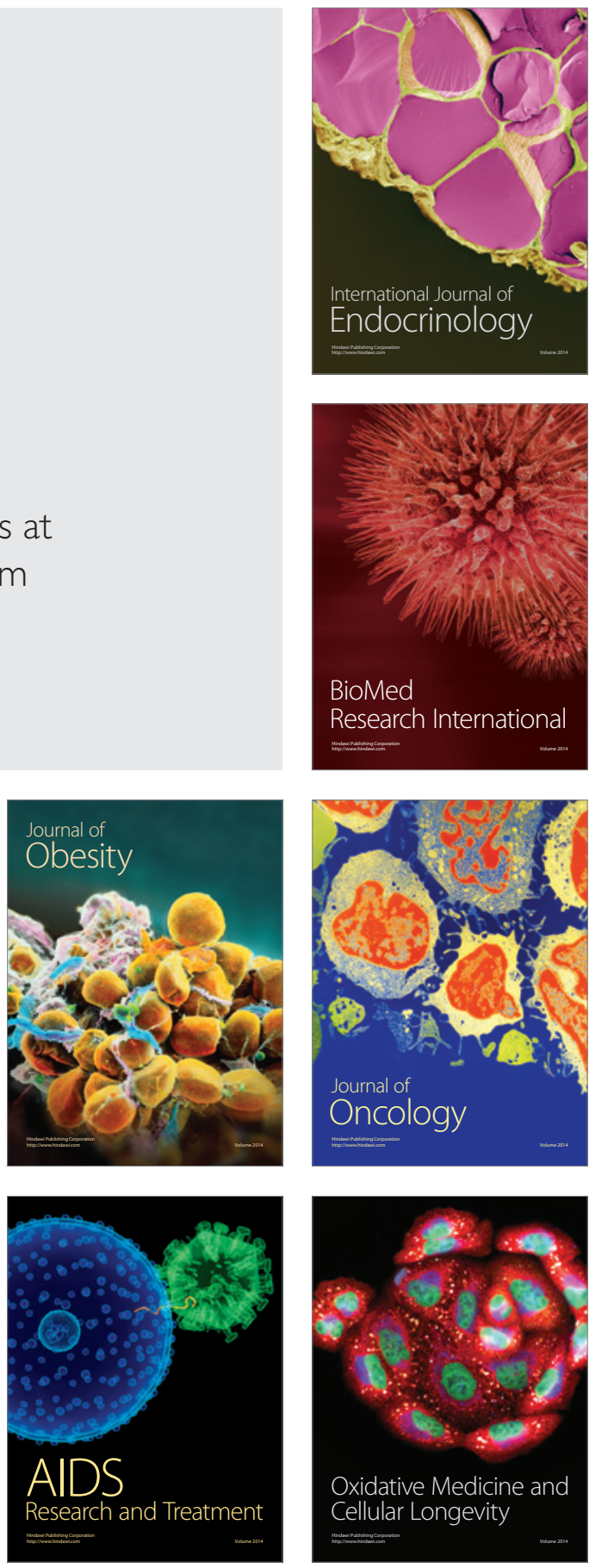